\title{
Endo-Urological Techniques for Benign Uretero-Ileal Strictures Have Poor Efficacy and Affect Renal Function
}

\author{
Elisabeth E. Fransen van de Putte ${ }^{a}$ Liesbeth L. de Wallc Erik van Werkhoven ${ }^{b}$ \\ Eddi A. Heldeweg ${ }^{c}$ Axel Bex ${ }^{a}$ Henk G. van der Poel ${ }^{a}$ Bas W.G. van Rhijn ${ }^{a}$ \\ Simon Horenblas ${ }^{\text {a }}$ Kees Hendricksen ${ }^{\text {a }}$ \\ ${ }^{a}$ Department of Urology, and b ${ }^{b}$ Department of Biometrics, The Netherlands Cancer Institute, and \\ 'Department of Urology, Onze Lieve Vrouwe Gasthuis West, Amsterdam, The Netherlands
}

\section{Keywords}

Balloon dilation · Stricture $\cdot$ Surgical anastomosis ·

Treatment · Urinary diversion

\begin{abstract}
Introduction: Uretero-ileal strictures (UES) following urinary diversion are therapeutically challenging. We compared the efficacy, safety and renal outcome following therapeutic endo-urological techniques (EUTs) and open surgical revision of the anastomosis (SRA) for UES. Material and Methods: We retrospectively analysed all EUTs and SRAs performed for UES in 2 hospitals between 1987 and 2015. Restenosis was defined as recurrent radiographically diagnosed hydronephrosis and re-intervention. Renal function (estimated glomerular filtration rate [eGFR]) decrease was correlated with the number of EUTs per patient. Results: Eighty-five UES were treated with 105 EUTs and 31 open revisions. Due to total obstruction, 28 (27\%) EUTs were aborted. During a median follow-up of 33 months, restenosis occurred following 53 out of 77 (69\%) completed EUTs and 4 out of 31 (13\%) SRAs ( $p<0.001$ on univariable and multivariable analyses). No serious (Clavien $\geq 3 \mathrm{~b}$ ) EUT-related complications occurred vs. $5(19 \%)$ related to SRA ( $p<0.001)$. The number of finalised EUTs was independently associated with eGFR loss
\end{abstract}

( $\beta=12.3 \mathrm{~mL} / \mathrm{min} / 1.73 \mathrm{~m}^{2}$ loss per EUT, $\left.p=0.008\right)$, with a significant cutoff value of $>1$ EUTs. SRA did not affect renal function ( $\beta=6.8 \mathrm{~mL} / \mathrm{min} / 1.73 \mathrm{~m}^{2}$ loss, $p=0.276$ ). Conclusions: Although EUTs are less invasive, they have an inferior efficacy to SRA. Our results suggest that a maximum of one EUT may be attempted without significantly compromising renal function.

(c) 2017 S. Karger AG, Basel

\section{Introduction}

Radical cystectomy is the standard treatment for muscle-invasive bladder cancer [1]. After the surgical removal of the bladder, a urinary diversion (UD) is created by anastomosing the ureters to a bowel segment, usually the terminal ileum.

Challenging complications following UD are benign strictures of the uretero-enteric anastomosis (UES). UES occur in 2.6 to $14 \%$ of patients [2-4] with a median postoperative time to diagnosis of $6-18$ months $[2,5,6]$. UES can lead to infections and renal function loss [7]. Classically, therapy consisted of open surgical revision of the anastomosis (SRA). Although the procedure is effective [8], postoperative complications are of a serious nature 
$[6,9]$. Over the years, less-invasive, endo-urological techniques (EUTs) have been developed.

Although EUTs are commonly used, their efficacy is scarcely investigated. Small, single-modality studies report success rates ranging from 16 to $88 \%[10,11]$. Comparative studies regarding EUT and SRA are limited. All report inferior efficacy for EUT [2, 6, 8, 9, 12-14]. However, it would be justified to consider EUT to delay or avoid open surgery, if the procedure is safe and does not compromise renal function.

In this 2-centre study, we compared long-term efficacy and safety of EUTs and SRA. Our secondary outcome was effective on renal function.

\section{Material and Methods}

\section{Patients}

We retrospectively screened 1,016 patients for UES. All patients had consecutively received a UD in either 1 of 2 hospitals (NCI: $n=834$ and OLVG: $n=182$ ) between June 1987 and January 2015 because of bladder cancer or tumours of other origin invading the bladder. All surgeries were performed by one team of the same surgeons. Uretero-ileal anastomoses were performed according to the refluxing Bricker technique [15]. From 1987 to 2012 , in case of ileal conduits and neobladders, the left ureter was anastomosed end-to-end and the right ureter end-to-side, whereas in pouch reservoirs, both anastomoses were end-to-side. Since 2012, both anastomoses were implanted end-to-side for all UDs. UES incidence was unknown for 66 patients (follow-up [FU] elsewhere or death $<3$ months $\mathrm{FU}$ ). Of the remaining 950 patients, 111 (11.7\%) were diagnosed with 139 UES (53 left-sided, 30 right-sided, 28 bilateral).

\section{Ureteral Strictures}

UES were diagnosed with antegrade pyelo-ureterography $(n=$ $107)$, intravenous pyelography-ureterography $(n=17)$, renal scintigraphy $(n=9)$ or retrograde ileo-ureterography $(n=6)$. Because of oncological reasons or comorbidity, 54 UES were left untreated or a double J or percutaneous nephrostomy tube was immediately accepted as a definitive solution. These UES were excluded from analysis. The remaining 85 UES were treated with one or several EUTs and/or SRA. Seven UES were immediately treated with SRA because of a failed attempt of EUT for a contralateral stenosis. All other UES were primarily treated with EUT. The decision to repeat EUT or switch to SRA was left to the discretion of the urologist.

\section{Treatment}

SRA comprised the resection of the affected distal ureter and reimplantation according to the Bricker technique (end-to-side) [15]. Used EUTs were antegrade or retrograde balloon dilation, sharp endo-ureterotomy or temporary stenting with a single J catheter. All procedures included subsequent temporary stenting with 8.5 Charriere single J catheters. The choice of EUT and duration of stenting was left to the discretion of the radiologist or urologist performing the procedure.

Repeated EUTs for Benign Uretero-Ileal Strictures Affect Renal Function
Treatment Outcome

FU of UES treatment included CT or ultra-sonography 2-6 weeks after stent removal. All patients subsequently had regular FU according to EAU guidelines [1], unless symptoms of restenosis occurred. Restenosis was defined as recurrent radiographically diagnosed hydronephrosis followed by re-intervention by means of at least nephrostomy tube replacement. Radioisotope renography was not performed if restenosis was obvious from hydronephrosis and renal function loss. We calculated stricture-free periods (SFP) from the date of stent removal to the date of the first sign of restenosis or the date of the last event-free FU. Renal function loss per patient was defined as the estimated glomerular filtration rate (eGFR) decrease from baseline (post-cystectomy) to the last cancer-free FU [16]. Patients for whom UES were left untreated following aborted EUTs were excluded from renal function analysis. Complications of completed procedures were scored according to the Clavien-Dindo Complication Classification system [17].

\section{Statistical Analysis}

Patient, stricture and treatment characteristics were compared using Mann-Whitney U test, 1-way ANOVA and Fisher's exact test. SFPs following EUT and SRA were plotted with the KaplanMeier method. A mixed effects Cox regression model was used with the treatment variables per stricture side as time-dependent covariates, and with a random intercept per patient to account for the fact that some patients developed 2 stricture locations (both left and right) [18]. The model included the following variables: patient American Society of Anesthesiologists score; history of pelvic radiotherapy; UD type; postoperative time to stricture diagnosis; side; presence of a contralateral stricture; stenting duration following treatment and the number of previous therapeutic procedures. Complications following EUT and SRA were compared using the Fisher's exact test. We investigated the association between the number of finalised EUTs per patient and eGFR loss using linear regression models, corrected for the presence of a contralateral stricture, adjuvant chemotherapy, time between the 2 eGFR measurements and additional performance of SRA of the anastomosis. Finally, restenosis and complications were compared between different EUT types with the Fisher's exact test. SPSS statistical software version 20.0 (SPSS Inc., Chicago, IL, USA) and R with package coxme (version 2.2-5) [18] were used for statistical analyses. $p$ values were considered significant at $<0.05$ on 2 -sided analysis.

\section{Results}

In total, 85 UES were treated in 70 patients with 136 procedures. Patient and stricture characteristics are shown in Table 1. The median postoperative time to diagnosis was 4.6 (range $0.2-85.1$ ) months. UES were predominantly left-sided and 17 patients had bilateral UES. Procedures consisted of 31 SRAs and 105 EUTs. Twentyeight (27\%) EUTs could not be completed due to total obstruction and were followed by immediate nephrostomy. These UES were subsequently treated with SRA ( $n=$ 28 ) or resolved with a permanent single $J$ catheter or nephrostomy tube $(n=15)$. The median number of final- 
Table 1. Baseline characteristics of the entire cohort. Patients were treated with a single endo-urological procedure (single EUT), multiple EUTs or with surgical revision of the anastomosis (either following EUTs or as primary treatment)

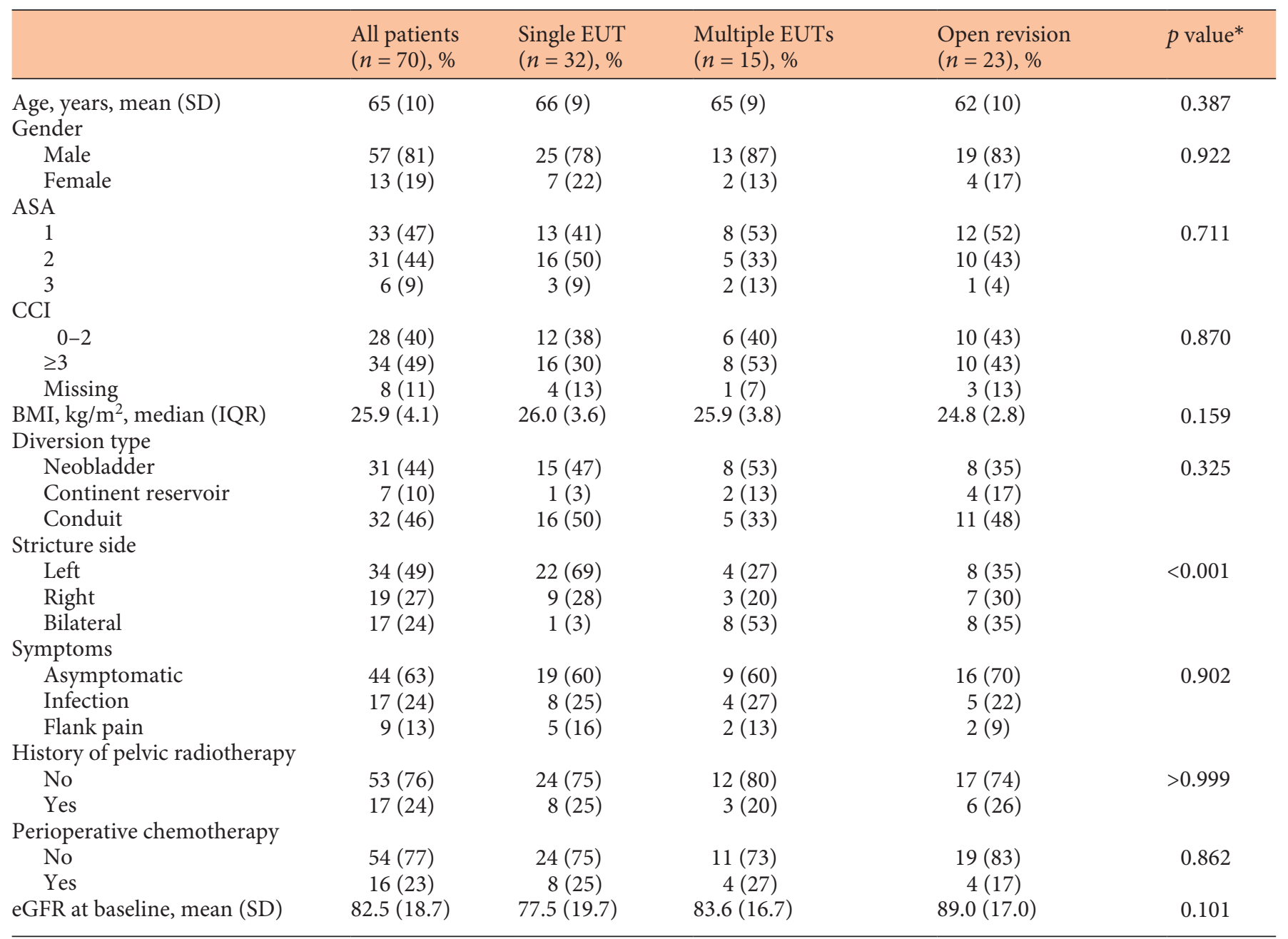

* $p$ value for comparison of all 3 groups.

EUT, endo-urological technique; ASA, American Society of Anesthesiologists score; CCI, age adjusted Charlson Comorbidity Index; $\mathrm{BMI}$, body mass index; eGFR, estimated glomerular filtration rate.

ised EUTs was 1 (range 0-6) per patient. Characteristics for the 108 completed procedures are shown in Table 2 . SRAs were more often performed after previously attempted unsuccessful interventions and the duration from diagnosis to treatment was longer than that for EUT. Additionally, the duration of subsequent stenting was shorter following SRAs. Other characteristics were comparable for both therapies.

Median FU for the 108 completed procedures was 33 (95\% CI 19-46) months. Restenosis occurred following 4 SRAs vs. 53 EUTs, resulting in intent-to-treat-based success rates of $87 \%$ for SRA vs. $23 \%$ for EUT $(p<0.001)$. Fourteen (24\%) restenoses were symptomatic (10\% infec- tion, $14 \%$ flank pain). SFP curves are displayed in Figure 1. Median SFP following EUT was 5 (95\% CI 0-10) months, while median SFP following SRA was not reached within the FU period $(p<0.001)$. This difference was also significant on multivariable analysis (hazard ratio: SRA vs. EUT $0.10, p<0.001)$. The risk of restenosis following EUT tended to be higher after pelvic radiotherapy (no radiotherapy vs. radiotherapy hazard ratio $0.57,95 \%$ CI $0.31-1.04, p=0.068)$ on multivariable analysis. None of the other included variables were predictive of restenosis following EUT or SRA (data not shown).

Baseline eGFR did not differ between patients who had received a single EUT, multiple EUTs or SRA (Table 1). 
Table 2. Stricture and treatment characteristics of completed procedures

\begin{tabular}{|c|c|c|c|c|}
\hline \multicolumn{5}{|l|}{ Stricture side } \\
\hline Left & $66(66)$ & $49(64)$ & $17(55)$ & \multirow[t]{2}{*}{0.263} \\
\hline Right & $42(39)$ & $28(36)$ & $14(45)$ & \\
\hline \multicolumn{5}{|l|}{ Contralateral stricture present } \\
\hline Yes & $46(42.6)$ & $30(39)$ & $16(52)$ & 0.162 \\
\hline \multicolumn{5}{|l|}{ Previous procedures } \\
\hline 0 & $69(64)$ & $62(81)$ & $7(23)$ & \multirow[t]{4}{*}{$<0.001$} \\
\hline 1 & $27(25)$ & $9(12)$ & $18(58)$ & \\
\hline 2 & $6(6)$ & $4(5)$ & $2(7)$ & \\
\hline 3 & $6(6)$ & $2(3)$ & $4(13)$ & \\
\hline \multicolumn{5}{|l|}{ Previous radiotherapy } \\
\hline Continent reservoir & $17(15.7)$ & $11(14.3)$ & $6(19)$ & \multirow{2}{*}{0.468} \\
\hline Conduit & $50(46.3)$ & $34(44.2)$ & $16(52)$ & \\
\hline \multicolumn{5}{|l|}{ Year of treatment } \\
\hline 1995-1999 & $14(13.0)$ & $12(15.6)$ & $2(6.5)$ & \multirow[t]{4}{*}{0.175} \\
\hline $2000-2004$ & $22(20.4)$ & $12(15.6)$ & $10(32.3)$ & \\
\hline 2005-2009 & $28(25.9)$ & $22(28.6)$ & $6(19.4)$ & \\
\hline $2010-2015$ & $44(40.7)$ & $31(40.3)$ & $13(41.9)$ & \\
\hline Postoperative time to diagnosis, months, median (IQR) & $5.0(8)$ & $5.3(9)$ & $4.6(8)$ & 0.783 \\
\hline Time from diagnosis to treatment, months, median (IQR) & $3.2(5)$ & $2.1(4)$ & $6.1(17)$ & $<0.001$ \\
\hline Temporary stenting duration, weeks, median (IQR) & $6.9(9)$ & $8.7(8)$ & $1.9(2)$ & $<0.001$ \\
\hline
\end{tabular}

* $p$ value for comparison of EUTs with open surgical revision. Previous procedures were all EUTs apart from 2 open surgical revisions of the anastomosis (1 prior to EUT and 1 prior to both EUT and open surgical revision).

EUT, endo-urological technique (endo-ureterotomy $n=3$, balloon dilation $n=61$, temporary stent $n=13$ ); IQR, interquartile range.

Fig. 1. Stricture-free periods (SFPs) following endo-urological techniques (EUTs) and open surgical revision of the anastomosis for benign uretero-ileal anastomotic strictures $(n=108, \log$ rank test $p<0.001)$.

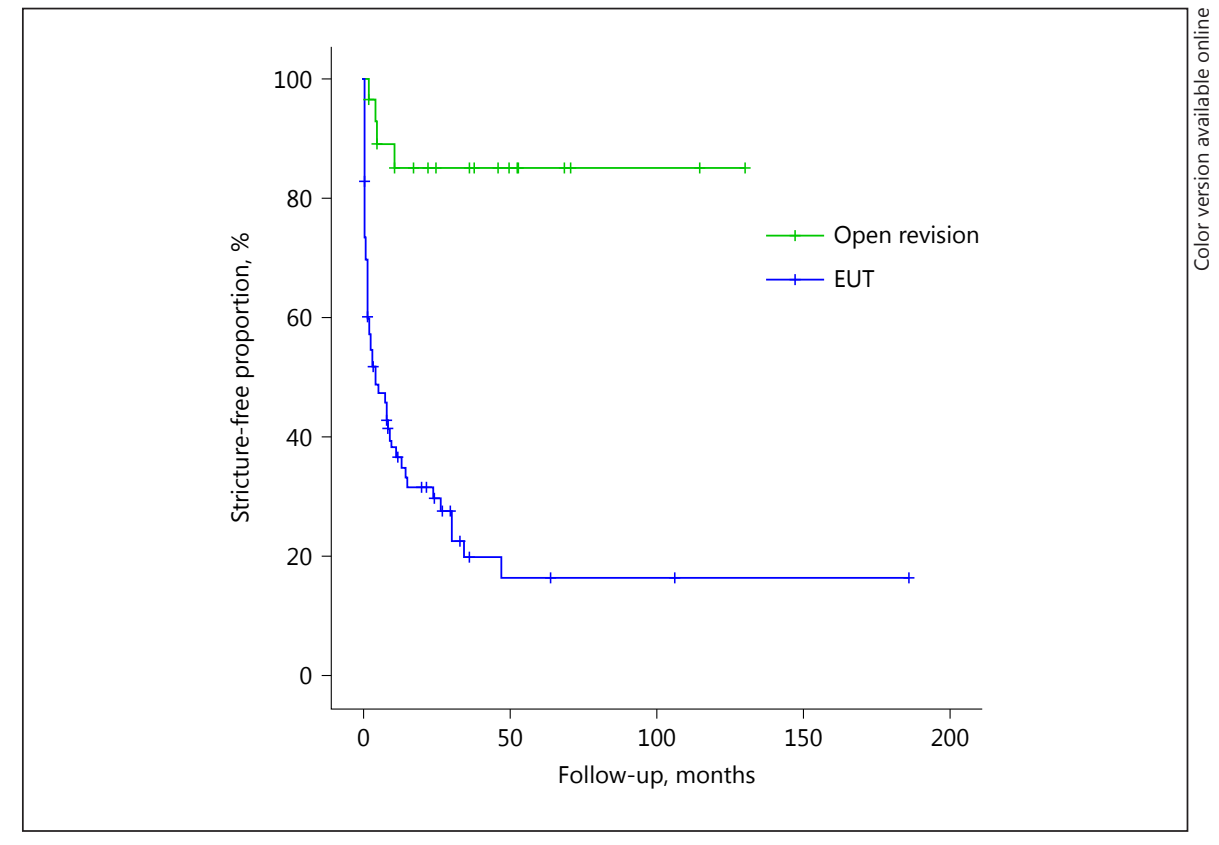

Urol Int 2018;100:18-24 
Renal function during the last disease-free FU (median 37 months, range 3-230) was available for 59 patients with finalized procedures. The mean eGFR decreased by $28.1(\mathrm{SD} 23.0) \mathrm{mL} / \mathrm{min} / 1.73 \mathrm{~m}^{2}$ for the entire cohort. The number of completed EUTs per patient was significantly associated with eGFR decrease on univariable (coefficient estimate $[\beta]=7.8 \mathrm{~mL} / \mathrm{min} / 1.73 \mathrm{~m}^{2}$ per EUT, SE 3.5, $p=0.028)$ and on multivariable analysis $(\beta=12.3 \mathrm{~mL} /$ $\mathrm{min} / 1.73 \mathrm{~m}^{2}$ per EUT, SE $4.5, p=0.008$ ). Cutoff values of $>1$ EUT per patient were associated with a significant eGFR decrease (Table 3). SRA, either following EUT or as a primary solution, did not affect renal function $(\beta=12.9$, SE 7.5, $p=0.090$ ).

Treatment safety was evaluable for 74 out of $77 \mathrm{com}-$ pleted EUTs and 27 out of 31 SRAs. EUTs induced $8(11 \%)$ Clavien 1-2 complications, while no Clavien $\geq 3 \mathrm{~b}$ complications occurred. Thirteen (48\%) complications occurred following SRAs (EUT vs. SRA, $p<0.001$ ), 8 were Clavien $1-2,3$ were Clavien $3 b$ ( 1 sigmoid perforation, 1 fascial dehiscence, 1 ileal anastomotic leakage) and 1 patient died of intestinal ischemia following bilateral SRA (Clavien 5).

Completed EUTs comprised 61 balloon dilations, 3 sharp endo-ureterotomies and 13 temporary stenting procedures. Strictures recurred after 42 (69\%) balloon dilations, 3 (100\%) endo-ureterotomies and $8(62 \%)$ temporary stenting procedures $(p=0.451)$. Neither the number of complications nor the loss in renal function differed significantly between EUT techniques (data not shown).

\section{Discussion}

EUTs are often performed as less invasive alternatives to SRA for UES. However, their therapeutic value has only been scarcely investigated. To our knowledge, this is the largest study to compare the efficacy, safety and renal outcome for both procedures. We found a $23 \%$ success rate for EUT (on intention-to-treat-based analysis) and $87 \%$ success rate for SRA of the anastomosis at a median FU of 33 months. These results are in line with efficacy reported in previous comparative studies (success-rate EUT $0-50 \%$, SRA 76-93\%) and confirm a significantly inferior efficacy for EUT [2, 6, 8, 9, 12-14]. Moreover, the short median SFP following EUT and the significant eGFR loss in case of restenosis indicate the necessity of careful FU and swift re-intervention.

Serious complications (Clavien $\geq 3 \mathrm{~b}$ ) occurred after $19 \%$ of SRAs. This high complication rate is not surpris-
Table 3.

a Association between estimated glomerular filtration rate (eGFR) decrease and the number of finalised endo-urological techniques (EUTs) as a continuous variable on multivariable analysis

\begin{tabular}{lcc}
\hline Variable & $\beta$ (SE) & $p$ value \\
\hline $\begin{array}{l}\text { Number of EUTs } \\
\text { Adjuvant chemotherapy (yes vs. no) }\end{array}$ & $12.3(4.5)$ & 0.008 \\
$\begin{array}{l}\text { Months between measurements } \\
\quad \text { continuous) }\end{array}$ & $0.12(0.06)$ & 0.069 \\
$\begin{array}{l}\text { Contralateral stricture present (yes vs. no) } \\
\begin{array}{l}\text { Open surgical revision performed (yes vs. } \\
\text { no) }\end{array}\end{array}$ & $-8.4(8.3)$ & 0.315 \\
& $12.9(7.5)$ & 0.090 \\
\hline
\end{tabular}

The coefficient estimate $(\beta) 12.3$ indicates that, each EUT is associated with a mean eGFR decrease of $12.3 \mathrm{~mL} / \mathrm{min} / 1.73 \mathrm{~m}^{2}$ after correction for the variables included in the table.

b Association between estimated glomerular filtration rate (eGFR) decrease and the number of finalised endo-urological techniques (EUTs) for different cut-off values on multivariable analysis*

\begin{tabular}{rcc}
\hline EUTs & $\beta(\mathrm{SE})$ & $p$ value \\
\hline 0 vs. $>0$ & $9.1(13.2)$ & 0.493 \\
$\leq 1$ vs. $>1$ & $18.4(8.1)$ & 0.027 \\
$\leq 2$ vs. $>2$ & $36.0(13.6)$ & 0.011 \\
\hline
\end{tabular}

* Corrected for: administration of adjuvant chemotherapy, time between eGFR measurements, presence of a contralateral stricture and performance of open surgical revision of the anastomosis yes/no. The coefficient estimate $(\beta)$ indicates the eGFR decrease in $\mathrm{mL} / \mathrm{min} / 1.73 \mathrm{~m}^{2}$ for each EUT cut-off value.

ing because of the invasive nature of the procedure and likelihood of the occurrence of intra-abdominal adhesions due to prior surgery, but it is higher than the reported rate in previous studies $(7-13 \%)[6,8,9,13,14]$. This might be explained by a relatively high number of patients with a history of pelvic radiotherapy [6]. However, small sample sizes and the absence of baseline patient characteristics in other reports prevent further comparison. We observed no serious adverse events following EUT. This suggests that, although less efficient, EUT is a safe initial option for UES. Moreover, neither SRA nor EUT efficacy was affected by the number of previously failed procedures.

However, the mean eGFR decreased by $28 \mathrm{~mL} /$ $\mathrm{min} / 1.73 \mathrm{~m}^{2}$ from baseline (post-cystectomy) to the last disease-free FU. Jin et al. [19] have previously investigat- 
ed renal function loss for 161 patients with UD. The median eGFR decrease was $8.5 \mathrm{~mL} / \mathrm{min}$ for conduit diversions and $2.0 \mathrm{~mL} / \mathrm{min} / 1.73 \mathrm{~m}^{2}$ for neobladders at 10 years FU. Urinary tract obstruction was an independent risk factor for renal function deterioration (eGFR decrease $>10 \mathrm{~mL} / \mathrm{min} / 1.73 \mathrm{~m}^{2}$ ) [19]. The relatively large eGFR decrease in our cohort confirms this finding. Furthermore, it reflects the consequences of poor EUT efficacy, as the number of completed EUTs per patient was independently associated with eGFR loss. The threshold for significant eGFR decrease was $>1$ EUT. This indicates that a maximum of 1 EUT per patient could be performed without compromising renal function. However, the actual number of EUTs that can be attempted should depend not only on statistical significance but also on the level of renal function that is acceptable to jeopardize for each patient.

We did not detect a difference in terms of efficacy between EUTs. The procedure most often performed in our cohort was balloon dilation, followed by temporary stenting without prior dilation. The largest series of balloon dilations thus far are reported by Schöndorf et al. [6] with a similar success rate of $25 \%$. The therapeutic value of temporary stenting without prior balloon dilation or incision can be disputed and has not been described in any previous report. Notably, in our cohort, treatment outcomes for temporary stenting were similar to those of balloon dilation. The question arises whether all diagnosed UES were true strictures, despite the fact that all were visualized by contrast imaging. We do not advocate the use of temporary stenting as a therapeutic measure based on these results. Rather, the similarly high re-stricture rate reflects the low efficacy of balloon dilation. Endo-ureterotomy has been described in a few reports as a slightly more effective procedure, especially when using a holmium:YAG laser (success rates 33$56 \%)[6,13,20]$. However, cohorts sizes were small (324 patients). In our series, only sharp endo-ureterotomy was applied. Although none of the endo-ureterotomy procedures was successful, the numbers in our series were too low to draw any conclusions regarding this procedure.

We did not identify predictive factors for treatment success, apart from a near-significant association between a history of pelvic radiotherapy and restenosis following EUT. Several retrospective studies suggest that a stricture length $>1 \mathrm{~cm}$ reduces the change of a successful EUT $[6,9,14]$. None had reported how stricture length was measured and whether this was done at the time of treatment or whether it was done retrospectively. We could not investigate stricture length, as it was documented only for a few patients at the time of treatment. Due to lack of a scale, retrospective measurement of stricture length is unreliable on contrast pyelo-uretergraphy.

Although a predictive role for stricture length seems plausible, prospective studies with uniform measurements are warranted to confirm this hypothesis.

Our study has several limitations. Due to the retrospective nature, a multitude of possible procedure-related issues, including surgeon and surgical techniques, could not be assessed. Also, the choice between repeating EUT and SRA was left to the discretion of the urologist. We acknowledge that this likely induced a selection bias. The fact that SRAs were more often performed after multiple previous interventions indicates that urologists were hesitant to perform open surgery. Although this was not associated with treatment outcome on multivariable analysis, a higher complexity of UES could have decreased SRA success rates. Likewise, EUT might be effective in a small subset of less severe UES. However, no definitive criteria to select these UES can be identified based on our results, nor on results in previous literature. Notwithstanding the above-mentioned limitations, the low EUT efficacy and their negative effect on renal function indicate that a more effective and safe therapy for UES is desirable. A maximum of 1 EUT can be performed per patient without significantly affecting their renal function.

\section{Disclosure Statement}

The authors have no conflicts of interest to declare.

\section{References}

1 Alfred Witjes J, Lebret T, Compérat EM, Cowan NC, De Santis M, Bruins HM, Hernández V, Espinós EL, Dunn J, Rouanne M, Neuzillet Y, Veskimäe E, van der Heijden AG, Gakis G, Ribal MJ: Updated 2016 EAU guidelines on muscle-invasive and metastatic bladder cancer. Eur Urol 2017;71:462-475.

2 Shah SH, Movassaghi K, Skinner D, Dalag L, Miranda G, Cai J, Schuckman A, Daneshmand S, Djaladat H: Ureteroenteric strictures after open radical cystectomy and urinary diversion: the university of Southern California experience. Urology 2015;86:87-91.

3 Vandenbroucke F, van Poppel H, Vandeursen H, Oyen R, Baert L: Surgical versus endoscopic treatment of non-malignant ureteroileal anastomotic strictures. Br J Urol 1993;71: 408-412. 
4 Madersbacher S, Schmidt J, Eberle JM, Thoeny HC, Burkhard F, Hochreiter W, Studer UE: Long-term outcome of ileal conduit diversion. J Urol 2003;169:985-990.

5 Anderson CB, Morgan TM, Kappa S, Moore D, Clark PE, Davis R, Penson DF, Barocas DA, Smith JA, Cookson MS, Chang SS: Ureteroenteric anastomotic strictures after radical cystectomy - does operative approach matter? J Urol 2013;189:541547.

6 Schöndorf D, Meierhans-Ruf S, Kiss B, Giannarini G, Thalmann GN, Studer UE, Roth B: Ureteroileal strictures after urinary diversion with an ileal segment - is there a place for endourological treatment at all? J Urol 2013; 190:585-590.

7 Minervini R, Pagni R, Mariani C, Morelli A, Morelli G, Minervini A: Effects on renal function of obstructive and nonobstructive dilatation of the upper urinary tract in ileal bladders with refluxing ureteroenteric anastomoses. Eur J Surg Oncol 2010;36:287-291.

8 Tal R, Sivan B, Kedar D, Baniel J: Management of benign ureteral strictures following radical cystectomy and urinary diversion for bladder cancer. J Urol 2007;178: 538-542.
9 Nassar OA, Alsafa ME: Experience with ureteroenteric strictures after radical cystectomy and diversion: open surgical revision. Urology 2011;78:459-65.

10 Shapiro MJ, Banner MP, Amendola MA, Gordon RL, Pollack HM, Wein AJ: Balloon catheter dilation of ureteroenteric strictures: long-term results. Radiology 1988;168:385387.

11 Yao K, Hou GL, Liu ZW, Yu SL, Li YH, Zhou FJ: Upper urinary tract dilation due to conglutination of intraluminal distal ureters after orthotopic bladder substitution with split-cuff nipple ureteral reimplants: early results of 8 cases. Urol Oncol 2009;27:611-616.

12 Msezane L, Reynolds WS, Mhapsekar R, Gerber G, Steinberg G: Open surgical repair of ureteral strictures and fistulas following radical cystectomy and urinary diversion. J Urol 2008; 179:1428-1431.

13 Laven BA, O'Connor RC, Gerber GS, Steinberg GD: Long-term results of endoureterotomy and open surgical revision for the management of ureteroenteric strictures after urinary diversion. J Urol 2003;170:12261230.

14 DiMarco DS, LeRoy AJ, Thieling S, Bergstralh EJ, Segura JW: Long-term results of treatment for ureteroenteric strictures. Urology 2001; 58:909-913.
15 Kouba E, Sands M, Lentz A, Wallen E, Pruthi RS: A comparison of the Bricker versus Wallace ureteroileal anastomosis in patients undergoing urinary diversion for bladder cancer. J Urol 2007;178:945-948.

16 Inker LA, Levey AS: Pro: estimating GFR using the chronic kidney disease epidemiology collaboration (CKD-EPI) 2009 creatinine equation: the time for change is now. Nephrol Dial Transplant 2013;28:1390-1396.

17 Dindo D, Demartines N, Clavien PA: Classification of surgical complications: a new proposal with evaluation in a cohort of 6336 patients and results of a survey. Ann Surg 2004; 240:205-213.

18 Therneau TM: Coxme: Mixed Effects Cox Models. R package version 2.2-5. 2015. https:// CRAN.R-project.org $/$ package $=$ coxme .

19 Jin XD, Roethlisberger S, Burkhard FC Birkhaeuser F, Thoeny HC, Studer UE: Long-term renal function after urinary diversion by ileal conduit or orthotopic ileal bladder substitution. Eur Urol 2012;61:491497.

20 Watterson JD, Sofer M, Wollin TA, Nott L, Denstedt JD: Holmium: YAG laser endoureterotomy for ureterointestinal strictures. J Urol 2002;167:1692-1695. 